\title{
Relation of atherosclerosis risk factors with the number of involved coronary arteries in angiography
}

\author{
Ali Golmohammadi ${ }^{1}$, Mohammadreza Taban Sadeghi ${ }^{1 *}$, Mina Bakhshayeshi ${ }^{1}$, Hossein \\ Namdar ${ }^{1}$, Ahmad Separham ${ }^{1}$
}

\begin{abstract}
Introduction: Coronary artery disease is the first leading cause of mortality in modern societies and formed the first cause of health expenditure. Male gender, diabetes mellitus, hypertension, hyperlipidaemia, family history of ischemic heart disease, personal history of ischemic heart disease, age, height, weight and smoking are the main risk factors for atherosclerosis and coronary artery diseases. Despite the abundant existing information about relation of these risk factors and atherosclerosis, there are different results regarding the relationship between these risk factors and the number of involved coronary arteries. The aim of this study is to determine the relation of these risk factors of coronary atherosclerosis with the number of involved coronary artery in angiography.
\end{abstract}

Material and Methods: In this cross-sectional study, a total of 300 patients during 8 months in ShahidMadani heart hospital were carried out as convenience sampling. Data was collected by questionnaire including age, sex, weight, height and body mass index, diabetes mellitus, hypertension, family history of coronary heart disease, smoking, drug addiction, occupation, place of residence and education were studied. Number of coronary arteries stenosis revealed by angiography. Data were analysed by software 17SPSS, Chi-square test, T test and ANOVA.

Results: A total of 300 patients with a mean age of $63.3 \pm 11.2$ year were enrolled. Collected data showed that $71 \%$ were male, $33.3 \%$ smokers, $57.3 \%$ hypertensive, $30 \%$ diabetic, $27.7 \%$ with hyperlipidaemia, $70.34 \%$ obese and $14 \%$ with a family history of heart disease. Frequency of one, two and three vessel involvement was respectively $30 \%, 32 \%$ and $38 \%$. There was a statistically significant relationship between ages, history of ischemic heart disease with the number of involved coronary artery. But there was no significant relationship with gender, body mass index, smoking and drug addiction, hypertension, family history of heart disease, location and education level with number of involved coronary artery.

Conclusions: Our study showed that despite the known role of conventional risk factors with the incidence and growth rate of atherosclerosis, but there is no direct correlation with some of these risk factors and the number of involved coronary arteries in coronary angiography

Key words: Coronary artery disease, Risk factors, Stenosis

\section{Introduction}

Coronary artery disease is one of the most common diseases of our era. It is one of the most important causes of mortality and morbidity in developed and developing countries (1). The World Health Organization predicts that in the next years in developing countries, the prevalence of the disease not only will not reduce but also until 2020 the mortality rate will reach 25 million per year (2).

Each year, people in the United States were diagnosed with coronary artery disease that annually causes the death of half a million people and this statistics is more than mortality of cancer, accidents and diabetes mellitus in this country $(3,4,5)$.
Cardiovascular disease, with a prevalence of $39 \%$ is considered the first cause of mortality in Iran. Studies showed between 900 to 1,200 people die each month in Tehran due to heart disease $(6,7,8)$.

Coronary artery disease is not only affecting mortality, but also can lead to disability, handicap, loss of productivity, poor quality of life; impose high expenditures and many social problems $(9,10)$. The costs associated with this disease in the United States is estimated about four hundred billion dollars (11). With increasing prevalence of coronary heart disease, factors that could affect incidence and mortality of these diseases come calling attention to the researchers. 
The results of this research led to a number of factors were known as cardiovascular risk factor including some social, environmental and behavioural factors that may increase the risk of atherosclerosis (12).

Framingham studies showed that coronary artery disease does not occur completely random and highrisk individuals can be detected based on number of some risk factors before the onset of clinical symptoms and concluded that a combination of these risk factors increased the risk of coronary heart disease (13). Hypercholesterolemia leads to atherosclerosis plaque formation and accumulation of these plaques cause narrowing of the coronary arteries and causes heart attack $(14,15)$. Cigarette smoking is one of the main preventable risk factors in the US and is allocated one-fifth of deaths from cardiovascular disease. Also, there are nearly 37 million deaths from cardiovascular disease in non-smokers due to contact with smokers (passive smokers). Clinically, smoking reduces high-density lipoprotein (HDL) and increases very low-density lipoprotein (VLDL) and blood glucose levels. After quitting smoking, the risk of cardiovascular disease can decrease up to 50 percent, and within 15 years the mortality due to cardiovascular disease will be similar to non-smokers $(16,17)$. The influence of drugs is not fully expressed in various studies, but some research showed that medication is effective on some risk factors for coronary heart disease (18).

Diabetes mellitus is one of the risk factors for cardiovascular disease (19). Diabetes is not only involved in the development of coronary artery disease, but also plays a role in various other diseases $(20,21)$. In many studies, hypertension is one of the risk factors on intensifying the coronary artery disease. Although, in some studies it has been rejected, but in general hypertension has been accepted as an important risk factor in coronary heart disease (22). Obesity, especially abdominal is a risk factor for cardiovascular disease and type 2 diabetes mellitus. Obese patients have glucose intolerance and insulin resistance more than thin or normal weight people. Patients with visceral obesity usually have disruption of plasma lipids and more risk of cardiovascular disease (23).

Other factors that increase the risk of cardiovascular diseases are aging and gender (male with a greater increase). Age of mortality of cardiovascular disease has decreased in the past few decades, so that the age of death in the United States in 2006 compared to 1950 for coronary artery disease, $69 \%$ and for ischemic heart disease, $76 \%$ decreased (24). There is a misconception that cardiovascular diseases often occur in men. Although before 60 decades the prevalence of cardiovascular disease is higher in men, after the $60 \mathrm{~s}$ and $70 \mathrm{~s}$ the prevalence of this disease is equal in both genders (25).

Medical Science and Discovery, 2016; 3(2): 65-70
Inflammation implicated in atherosclerosis and acute coronary syndromes and atherosclerosis is an inflammatory disease. White blood cells play an important role in the onset and progression of atherosclerosis (26). Also, along with risk factors the average of white blood cells increases significantly (27).

According to items listed above and due to different cultures, social environments and living conditions, the prevalence of risk factors for coronary artery disease have been different. Identifying the risk factors of this disease has great importance to health programming. Furthermore, any plan for the prevention of coronary artery disease requires sufficient information about its epidemiology and identification associated of high risk groups and factors. So, the aim of this study is to find the relationship between risk factors for coronary artery disease with the number of coronary artery stenosis in angiography for proper planning based on the results to reduce causes comes into action.

\section{Material and methods}

This cross- sectional study was carried out for eight months in ShahidMadani heart hospital as convenience sampling. The study population included 300 patients with coronary artery disease which was confirmed by coronary angiography and were hospitalized in ShahidMadani heart hospital. Data was collected by questionnaire including age, sex, weight, height and body mass index, diabetes mellitus, hypertension, family history of heart disease, smoking, drug addiction, occupation, place of residence and education. Number of stenotic arteries was revealed by angiography. Data were analyzed by Chi-square test, $\mathrm{T}$ test and ANOVA with software 17SPSS. $P$ value equal to or less than 0.05 was considered statistically significant.

\section{Results}

Of the total 300 patients included in this study, 213 patients were male $(71 \%)$ and 87 patients were female (29\%). The mean age of participants were $63.3 \pm 11.2$ years $(\operatorname{Min}=25$ years and $\operatorname{Max}=84$ years). The mean age of women was significantly higher than the mean age of men $(\mathrm{P}=0.04) .42$ patients $(14 \%)$ had a positive family history of ischemic heart disease. Mean left ventricle Ejection Fraction of patients were 47.6 \pm 8.9.

Out of, 300 patients, 87 admitted with ST elevation myocardial infarction, 35 patients with Non ST elevation MI, 124 patients with unstable Angina, 49 patients with chronic stable Angina and 5 patients due to positive exercise test or heart scan underwent coronary angiography (Figure 1).

In our study, $57.3 \%$ of patients had a history of hypertension, $33.3 \%$ had a history of smoking, $1.7 \%$ had a history of opiate drug addiction, $0.7 \%$ had a history of alcohol and $1 \%$ had a history of smoking shisha. The average body mass index (BMI) of the 
patients were $27.7 \pm 4.9$ ( $\mathrm{Min}=16.4$ and $\mathrm{Max}=54.9)$ ). Incidence of three, two and one vessel involvement did not differ among women and men $(\mathrm{P}=0.07)$. Most coronary artery involvement related to three vessels disease by $38 \%$ and 14 patients $(4.67 \%)$ had involvement of left main coronary lesion. The age of patients with the number of coronary artery involvement had direct and significant correlation $(\mathrm{P}=0.02)$.
After examining the relationship of the past medical history with the number of stenotic coronary arteries, we conclude that just in patients with a history of ischemic heart disease there were significant differences among the groups with one, two and three vessel involvement $(\mathrm{P}<0.001)($ Table 1$)$. There were no significant correlations among the other past medical history with the number of stenotic coronary arteries. Left ventricular ejection fraction had direct and significant correlations with number of involved coronary arteries. (Table 2 ).

\begin{tabular}{|llcccc|}
\hline \multicolumn{1}{|c|}{ Table 1: Qualitative data of patients based on the number of 1 Vessel involved } \\
& \multicolumn{1}{c}{ Categories } & $\begin{array}{c}\text { 1 Vessel } \\
\text { involved }\end{array}$ & $\begin{array}{c}\text { 2 Vessel } \\
\text { involved }\end{array}$ & $\begin{array}{c}\text { 3 Vessel } \\
\text { involved }\end{array}$ & P- Value \\
& Male & $65(30.5 \%)$ & $75(35.2 \%)$ & $73(34.3 \%)$ & 0.07 \\
\hline Gender & Female & $25(28.7 \%)$ & $21(24.1 \%)$ & $41(47.1 \%)$ & \\
\hline & Illiterate & $35(32.7 \%)$ & $29(27.1 \%)$ & $43(40.2 \%)$ & \\
\cline { 2 - 6 } Education & Undergraduate & $39(29.3 \%)$ & $43(32.3 \%)$ & $51(38.3 \%)$ & \\
\cline { 2 - 6 } & Diploma & $8(20.5 \%)$ & $19(48.7 \%)$ & $12(30.8 \%)$ & 0.18 \\
\cline { 2 - 6 } & Bachelor & $4(66.7 \%)$ & $1(16.7 \%)$ & $1(16.7 \%)$ & \\
\cline { 2 - 6 } & Higher education & $1(50 \%)$ & $1(50 \%)$ & $0(0 \%)$ & \\
\hline \multirow{2}{*}{ Resident } & Urban & $70(29.5 \%)$ & $77(32.5 \%)$ & $90(38 \%)$ & 0.92 \\
\hline Blood pressure & Rural & $20(31.7 \%)$ & $19(30.2 \%)$ & $24(38.1 \%)$ & \\
\hline Diabetes mellitus & Yes & $43(25 \%)$ & $59(34.3 \%)$ & $70(40.7 \%)$ & 0.09 \\
\hline \multirow{2}{*}{ Hyperlipidemia } & Yes & $20(22.2 \%)$ & $29(32.2 \%)$ & $41(45.6 \%)$ & 0.15 \\
\hline Ischemic heart disease & Yes & $26(31.3 \%)$ & $29(34.9 \%)$ & $28(33.7 \%)$ & 0.69 \\
\hline Positive family history & Yes & $10(14.3 \%)$ & $25(35.7 \%)$ & $35(50 \%)$ & \\
\hline Smoking & Yes & $13(31 \%)$ & $13(31 \%)$ & $16(38.1 \%)$ & 0.98 \\
\hline
\end{tabular}

Table 2: Quantitative of patients based on the number of Vessel involved

\begin{tabular}{|lcccc|}
\hline \multicolumn{1}{r}{ Variables } & 1 Vessel Disease & 2 Vessel Disease & 3 Vessel Disease & P-Value \\
\hline BMI & $27.85 \pm 5.04$ & $27.96 \pm 5.35$ & $27.44 \pm 4.64$ & 0.72 \\
\hline EF (\%) & $49.11 \pm 9.46$ & $48.02 \pm 7.04$ & $45.95 \pm 9.39$ & $0.03^{*}$ \\
\hline Age & $58.85 \pm 12.73$ & $65.65 \pm 12.12$ & $63.29 \pm 10.11$ & $0.02^{*}$ \\
\hline
\end{tabular}

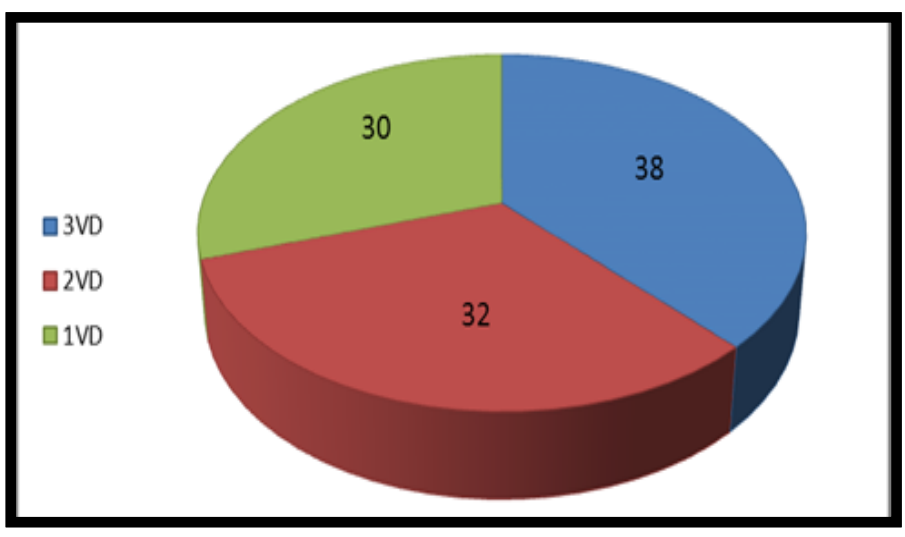

Figure 1. Frequency of the number of Vessel involved. 1VD: 1 Vessel Disease; 2VD: 2 Vessels Disease; 3VD: 3 Vessels. Disease Data are represented as \% of involvement. 


\section{Discussion}

This study showed that age has a direct impact on the number of involved coronary arteries confirmed by coronary angiography and by increasing age, number of involved coronary arteries increase too. Several previous studies described the relationship between cardiovascular risk factors with severity of coronary artery involvement and shown that patient with abnormal angiography had a higher mean age than people with normal angiography (28). Studies have also showed that, by increasing age, coronary artery involvement increases too (29-32).

Our study also showed that gender had no effect on the number of involved coronary arteries. The impact of gender on the number of coronary arteries involved has been different and some studies have reported similar results (30). But in some studies the number of coronary arteries involved in women was higher than men (33). While the results of some studies have reported that the number of coronary arteries involved in men was higher than women $(29,34)$, in our study, ineffectiveness of gender in the number of coronary arteries involved may be due to older women in the study group compared to men.

In our study, BMI was not associated with the number of involved vessels. Similar results were obtained in other studies too (37). In several studies, the average body mass index with the number of vessels involvement has been shown significant difference $(20,35,36)$. In our study, due to no difference between the sets of data, there may be higher prevalence of obesity in the younger population than in previous decades and so neutralizes the effect of it through the impact of age on the number of involved vessels.

Also, there was not a statistically significant relationship between hypertension and the number of involved vessels. In previous studies, it has been shown that hypertension associated with a greater coronary artery disease $(38,39)$. Despite of proved relation among hypertension, atherosclerosis and coronary artery disease, other studies have not shown relationship between hypertension and number of involved vessels (40).

In this study, smoking and drugs addiction and alcohol use were not significantly associated with the number of occluded coronary arteries. In spite of relevance of cigarette smoking, drug addiction and alcohol use with atherosclerosis, some other studies have not shown the relationship between these factors and the number of involved vessels $(1,29,31$ and 40). Due to the increase in Cigarette use in youth and starting at a younger age, it can be adjusted with age factor. Disaffiliation of the number of involved vessels with alcohol also may be due to the small number of patients taking alcohol. It has been shown that drug addiction more than two years can be a risk of coronary artery stenosis (41).
Moreover, there was no significant association between diabetes mellitus and number of involved coronary artery (according to the classification in one, two and three vessel), but there was a possible significant difference if it was analyzed based on one vessel and more than one vessel. In other studies it has been shown that diabetics are more prone to multi vessel coronary disease (42).

There was not significant correlation between history of diagnosed hyperlipidemia before admission and the number of affected coronary arteries. However, similar results were obtained in some studies (29). However, other studies have shown that increase of low-density lipoprotein is a risk factor of severity and number of coronary arteries involvement (43). In our study cause of no relationship between hyperlipidemia and number of involved coronary artery may be use of history of hyperlipidemia based on presence or absence of diagnosed disease before admission but other studies asses' relationship between serum Lowdensity lipoprotein and cholesterol level and severity of coronary artery involvement.

Left ventricular ejection fraction was different in the three groups and had a significant relationship with the number of stenotic coronary arteries $(\mathrm{P}=0.03)$. Previous studies showed there was significant relationship between Left ventricular ejection fraction and the number of involved coronary arteries (29). In our study, there was no significant relationship among their level of education, place of residence and occupation with the number of stenotic coronary arteries. A study has shown similar results (35). In contrast, a different study showed significant association between these factors and the number of affected coronary arteries (29).

\section{Conclusion}

In this study, age had a direct relation with number of coronary artery stenosis in angiography $(\mathrm{P}=0.02)$. There was no significant difference between men and women in the number of involved vessels $(\mathrm{P}=0.07)$. Other known risk factors of atherosclerosis had no statistically significant correlation with the number of coronary arteries stenosis. There was a significant relation between the left ventricular ejection fractions of patients with number of involved coronary artery.

Conflict of Interest: The authors declare no potential conflicts of interest with respect to the research, authorship, and/or publication of this article.

\section{References}

1. ZandParsa AF, Ziai H \&Fallahi B. The relationship between cardiovascular risk factors and the siteand extent of coronary artery stenosis during angiography, School of Medicine of Tehran Medical Sceiences Journal 2010; 68(3): 182-7. 
2. Lukkarinen, H., Hentinen, M. Treatments of coronary artery disease improve quality of life in the long term, Nurs Res 2006; 55: 26-33

3. Martin C, Martin A. Mortality Trends in Cardiovascular Disease: a New Model to Visualise the Contribution of Specific Diseases, Cohort Effects and Coding Changes to Overall Mortality Improvement. Value Health. 2015 Nov;18(7):A383.

4. Ordunez P, Prieto-Lara E, Pinheiro Gawryszewski V, Hennis AJ, Cooper RS. Premature Mortality from Cardiovascular Disease in the Americas - Will the Goal of a Decline of " $25 \%$ by 2025 " be Met? PLoS One. 2015 Oct $29 ; 10(10)$

5. Rodrigues PC, Santos ES, Ignotti E, Hacon SS. SpaceTime Analysis to Identify Areas at Risk of Mortality from Cardiovascular Disease. Biomed Res Int. 2015;2015:841645.

6. KariminejadA.Investigate thegeneticpolymorphism ofACEas a cause ofcardiovascular diseasein thepopulation, genetic2010;8(1): p1(Persian).

7. Sadegi M. Prevalence ofcoronary artery diseasein centralIranAriaJournal 2006; 6(35):70-74. (Persian).

8. Sarraf-Zadegan N, Boshtam M, Malekafzali H Bashardoost N, Sayed-Tabatabaei FA, Rafiei M, Khalili A, et al. Secular trends in cardiovascular mortality in Iran, with special reference to Isfahan. Acta Cardiol. 1999 Dec;54(6):327-33.

9. Allen NB, Badon S, Greenlund KJ, Huffman M, Hong Y, Lloyd-Jones DM. The association between cardiovascula health and health-related quality of life and health status measures among U.S. adults: a cross-sectional study of the National Health and Nutrition Examination Surveys, 20012010. Health Qual Life Outcomes. 2015 Sep 22;13(1).

10. Rodrigues GH, Gebara OC, Gerbi CC, Pierri H, Wajngarten M. Depression as a Clinical Determinant of Dependence and Low Quality of Life in Elderly Patients with Cardiovascular Disease. Arq Bras Cardiol. 2015 Jun;104(6):443-9.

11. Gazianojm, LibbyP, Bonow Ro. Globa burden of cardiovascular disease.Braunwalds Heart Deases 2005; 7:423-55.

12. Pourmand K, Sadeghi M, Sanei H, Akrami F, Talaei M.2007. Which major atherosclerosis' risk factor represents the extent of coronary artery disease?, J Isfahan Med Sch ,25: 61-71.

13. Scott MG, Gary JB, Michael HC. Primary prevention of coronary heart disease: Guidance from Framingham. Circulation 199; 97:1876-87.

14. Grundy SM, Bilheimer D, Blackburn H. Rationale of the diet-heart statement of the American heart association. Circulation $1982 ; 65(4): 839-54$.

15. Kartz M. Dietary cholesterol, atherosclerosis and coronary heart disease.HandbExp Pharmacol2005; 170:195- 213.

16. American Heart Association. 1998. Heart and stroke statistical update heart and stroke statistical update. Texas: American Heart Association.

17. Fellows JL, Trosclair A. Annual smoking-attributable mortality, years of potential life lost and economic costs:United States 1995-1999, MMWR Morb Mort Wkly, 2002 ;51(14):300-3.

Medical Science and Discovery, 2016; 3(2): 65-70 http://dx.doi.org/10.17546/msd.23422

18. Asgary S, Sarrafzadegan N, Naderi GA, Rozbehani R. Effect of opium addiction on new and traditional cardiovascular risk factors: do duration of addiction and route of administration matter. Lipids Health Dis 2008; 7 : 42.

19. Molstad P. 2007. Coronary heart disease in diabetics: prognostic implications and results of interventions. Scand Cardiovasc J , 41: 357-362

20. Pourmand K, Sadeghi M, Sanei H, Akrami F, Talaei M. Which major atherosclerosis' risk factor represents the extent of coronary artery disease?, J Isfahan Med Sch 2007; 25: 61-71.

21. Molstad P. Coronary heart disease in diabetics: prognostic implications and results of interventions. ScandCardiovasc J 2007; 41: 357-362.

22. Jankowski P, Kawecka-Jaszcz K, Bilo G, PajakA. Determinants of poor hypertension management in patients with ischaemic heart disease. Blood Press 2005; 14: 284292.

23. Saedi M, AkhavaTabib A, Jokar MH, Yazdani A Prevalence of cardiovascular risk factors in male individuals with hypertriglecamic waist phenotype. Med J MashadUniv Med Scince 2007; 50: 259-268.

24. Murray CJL, Lopez AD.. Alternative projections of mortality and disability by cause 1990-2020: Global Burden of Disease Study. Lancet 1997; 349:1498-1504.

25. Kreatsoulas C, Natarajan MK, Khatun R, Velianou JL, Anand SS. Identifying women with severe angiographic coronary disease. J Intern Med 2010;268: 66-74.

26. Elkind MS, Sciacca R, Boden-Albala B, Homma S, Di Tullio MR. Leukocyte count is associated with aortic arch plaque thickness. Stroke 2002; 33(11): 2587-92.

27. Zeltser D, Rogowski O, Fusman R, Rotstein R, Rubinstein A, Koffler M, et al. The multiplicity of atherosclerotic risk factors corresponds to the appearance of increased leukocyte count in the peripheral blood relevance to the pathogenesis of the disease, JCardiovasc Risk 2001; 8 (6): 37982

28. Humphries $\mathrm{KH}, \mathrm{Pu} \mathrm{A}$, Gao M, Carere RG \&Pilote L. Angina with "normal" coronary arteries: Sex differences in outcomes. American Heart Journal 2008; 155(2): 375-81.

29. Masoomi M \&Nasri HR. Relationship between coronary risk factors and the number of involved vessels in coronary angiography. Journal of Hormozgan University of Medical Sciences 2006;10(1): 29-34

30. Bigi R, Cortigiani L, Colombo P, Desideri A, Bax JJ,Parodi O. Prognostic and clinical correlates of angiographically diffuse non-obstructive coronary lesions. Heart 2003; 89: 1009-1013.

31. Darabian S, Abbasi A. The correlation of ischemic risk factors with left main tract disease.Feyz2007; 11:31-35.

32. Veeranna V, PradhanJ, Niraj A, Fakhry H, Afonso L. Traditional cardiovascular risk factors and severity of angiographic coronary artery disease in the elderly. prevcardiol 2010;13: 135- 140 .

33. Hochner-Celnikier D, Manor O, Gotzman O, Lotan H, Chajek-Shaul T. Gender gap in coronary arteryndisease: comparison of the extent, severity and risk factors in men and women aged 45-65 years. Cardiology2002;97(1):1823 
34. Kreatsoulas C, Natarajan MK, Khatun R, Velianou JL \&Anand SS. Identifying women with severe angiographic coronary disease. Journal of Internal Medicine 2010;268(1): 66-74

35. HosseiniA, Abdullah A, Behnam pour N, Salehi A. peace be upon him, calledpornography, Relationship betweencardiovascular risk factorsandvasculardiseasebased on theresults ofangiography, ParamedicalFaculty ofTehranUniversity of Medical Sciences (Payavardhealth) 2011;6(5):391-383.

36. Niraj A, Pradahan J, Fakhry H, Veeranna V \&Afonso L Severity of Coronary Artery Disease in Obese Patients Undergoing Coronary Angiography: Obesity Paradox Revisited. ClinCardiol 2007; 30(8): 391-6

37. Auer J, Weber T, Berent R, Lassnig E, Maurer E, Lamm G, et al. Obesity, body fat and coronary atherosclerosis International Journal of Cardiology 2005; 98(2): 227-35.

38. Guo YH, Zhang WJ, Zhou YJ, Zhao D, Zhou ZM, Zhang H. Study of the relationship between cardiovascular risk factors and severity of coronary artery disease in patients underwent coronary angiography. Zhonghua Xin Xue Guan Bing Za Zhi. 2005 May;33(5):415-8. http://dx.doi.org/10.17546/msd.23422

39. Veeranna V, PradhanJ, Niraj A, Fakhry H, Afonso L. Traditional cardiovascular risk factors and severity of angiographic coronary artery disease in the elderly. prev cardiol, 2010. 13: 135- 140.

40. Aygul N, Ozdemir K, Abaci A, Aygul MU, Duzenli MA, Yazici HU, et al. Comparison of Traditional Risk Factors, Angiographic Findings, and In-Hospital Mortality between Smoking and Nonsmoking Turkish Men and Women With Acute Myocardial Infarction. ClinCardiol $2010 ; 33(6)$ : $49-54$.

41. Sadeghi A, Nizal S, Naderi Gh. A, RozbehaniR. Effect of opium addiction on new and traditional cardiovascular risk factors: do duration of addiction and route of administration matter? Lipids in Health and Disease 2008; 7(42):1-5.

42. Uddin SN, Malik F, Bari MA, Siddiqui NI, Khan GK, Rahman S., et al. 2005. Angiographic severity and extent of coronary artery disease in patients with type 2 diabetes mellitus. Mymensingh Med J ,14(1):32-7.

43. Hong MK, Romm PA, Reagan K, Green CE, Rackley CE. Usefulness of the total cholesterol to high-density lipoprotein cholesterol ratio in predicting angiographic coronary artery disease in women.Am J Cardiol 1991; 68(17):1646-50.

Copyright (C) 2014 The Author(s); This is an open-access article distributed under the terms of the Creative Commons Attribution License (http://creativecommons.org/licenses/by/4.0), which permits unrestricted use, distribution, and reproduction in any medium, provided the original work is properly cited. All Rights reserved by international journal of Medical Science and Discovery. 